\title{
Multilevel Resistive Switching and Nonvolatile Memory Effects in Epoxy Methacrylate Resin and Carbon Nanotube Composite Films
}

\author{
Yanmei Sun ${ }^{\text {a,b }}$, Junguo Lu ${ }^{\text {a,b }}$, Chunpeng Ai ${ }^{a}$, Dianzhong Wen ${ }^{\text {a,* }}$, Xuduo Bai ${ }^{\text {c,* }}$ \\ ${ }^{\mathrm{a}}$ HLJ Province Key Laboratories of Senior-education for Electronic Engineering, Heilongjiang University, \\ Harbin,150080, China \\ ${ }^{\mathrm{b}}$ Communication and Electronics Engineering Institute, Qiqihar University, Qiqihar,161006, China \\ ${ }^{\mathrm{c}} \mathrm{School}$ of Chemistry and Materials Science, Heilongjiang University, Harbin,150080, China \\ E-mail: sym791122@163.com*
}

ABSTRACT: A novel multilevel resistive switching was observed in epoxy methacrylate resin (EMAR) and carbon nanotubes (CNTs) composite films fabricated by spin coating method. The fabricated devices demonstrated the rewritable nonvolatile memory characteristics. More significantly, the memory device based on EMAR+CNTs composite exhibits multilevel stable conductivity states with stable intermediate resistance states in response to the applied voltage. By setting different compliance current and content of CNTs in composite film, the multilevel ON-states and even the multilevel OFF-states have been observed in our memory device. As fabricated devices exhibited multilevel resistive switching with stable resistance ratio between different resistance states having good data retention and endurance characteristics. It offers a novel design strategy for solution processable multilevel data storage.

KEYWORDS: Resistive switching, multilevel memory, carbon nanotubes, rewritable, charge trap

\section{Introduction}

Organic resistive memory devices have attracted plenty of attention in recent years [1-8], due to the conventional flash memory approaching its scaling limits. Organic materials have exhibited a lot of advantages, including good processability [9], flexibility [10-12], light weight, low cost [13], and the possibility for molecular design through chemical synthesis 
$[14,15]$, which are promising candidate for developing next-generation memory devices. Resistive memory devices primarily take advantage of reversible resistive switching between the different resistance states to write/erase the user's information [16,17]. Up to now, most organic resistive memory devices are binary systems comprised by two conductivity states (i.e., ON and OFF states) [18-23]. An increasing number of demands for high density data storage have stimulated a particular research attention to multilevel resistive switching memory [24-27]. The multilevel tunability of resistivity provides a unique opportunity to store more than 2-bits in a single cell, making possible achieving a high density memory with minimized downscaling.

Among various resistive memory devices, the nanocomposite resistive memory comprised by charge trapping conductive materials, such as metal nanoparticles [28-30], fullerene [31], PCBM [32], graphene [33,34], or carbon nanotubes (CNTs) [35-39], dispersed in a polymer matrix was attached a great deal of attention. The synergistic combination of the polymer matrix and the nanomaterials with strong electrical properties offers plentiful of advantages, such as facile solution processing, high-mechanical flexibility, and the tunability of resistive switching with material composition. For the sake of the successful fabrication of reliable nanocomposite resistive memory, uniform dispersion of charge trapping materials in a polymer matrix is crucial.

CNTs exhibit unique electronic, mechanical, and chemical properties that make them attractive for memory devices [40]. CNTs undergo covalent functionalisation during the dispersion in a polymer matrix [41]. A main shortcoming of covalent functionalisation is the disruption of the extended $\pi$-conjugation in the CNTs. This has a negative impact on the electrical properties since each covalent functionalisation site scatters electrons. Therefore, polymeric composites with CNTs are an alternate method for CNTs. In the last years several attempts have been made to mix CNT with polymeric materials, G. Liu et al. found that 
through controlling the CNT content in the PVK-CNT composite film, electronic devices with a sandwich structure of ITO/PVK-CNT/Al are capable of exhibiting insulator behavior, bistable electrical switching behavior (WORM memory and rewritable memory effects), and conductor behavior [35]. Later, by blending various weight percentages of CNT in PVA, Pandurangan et al. suggested the characteristic behavior of an insulator, WORM, flash, and conductor effect is exhibited once again [39]. Moreover, CNTs can improve the performance of WORM memories by doping the F12TPN layer with about 1 wt $\%$ of carbon nanotubes [37]. Later, I. A. Hümmelgen et al. suggested the volatile bistable memory behavior at a small quantity $(\leq 0.01 \mathrm{wt} \%)$ of CNTs embedded in a PEDOT: PSS conducting polymer matrix [38]. Although polymeric composites with CNTs has been extensively studied, to the best of our knowledge, its multilevel resistive switching behaviors have not been reported yet.

In the present study we have utilized a insulating polymer, epoxy methacrylate resin (EMAR) to fabricate an EMAR+CNTs composite layer for the memory device. We have chosen EMAR as the basic matrix due to the following advantages; it is easily processed, has a good film forming and good adhesive nature for applications in electronic devices. Furthermore, it is considered a excellent host matrix for CNTs. On account of the good dispersion of CNTs in EMAR, compared to other polymer matrices, the functionalisation of the CNTs can be avoided. In this work, we report multilevel switching resistive memory fabricated from the facile solution casting of EMAR mixed multiwalled CNTs composites. The fabricated indium tin oxide (ITO)/EMAR+CNTs/Al devices demonstrated the rewritable nonvolatile memory characteristics with good data retention and endurance characteristics. More significantly, the memory device based on EMAR+CNTs composite exhibit multilevel stable conductivity states with stable intermediate resistance states in response to the applied voltage. By setting different compliance current and content of CNTs in composite film, the 
multilevel ON-states and even the multilevel OFF-states have been observed in our memory device.

\section{Experimental section}

2.1. Materials. The EMAR $\left(M_{\mathrm{w}} \sim 2400\right)$ was purchased from Sigma-Aldrich. Carboxylmultiwalled CNTs were purchased from NanoLab Inc. (Beijing, China). The outer diameter, length, specific surface area, and purity of the CNTs were $5-11 \mathrm{~nm}, 2-10 \mu \mathrm{m}, 233 \mathrm{~m}^{2} / \mathrm{g}$, and 95\%, respectively. Transmission electron microscopy (TEM) measurements were carried out to observe the micro-structural properties of the CNTs, as shown the inset in Fig. 1a.

2.2. Device Fabrication and Characterization. The ITO glass of dimensions $2 \mathrm{~cm} \times 1$ $\mathrm{cm}$ (sheet resistance $R_{\square}=6-9 \Omega / \square$ ) used for memory device was precleaned by ultrasonication with deionized water, acetone, isopropanol and ethyl alcohol each for 20 min. The memory device was fabricated with the configuration of ITO/EMAR+CNTs/Al. The blends with different mixing ratio were prepared by the acetone solution of EMAR $(5 \mathrm{mg} / \mathrm{ml})$ blending with $5 \mathrm{mg} / \mathrm{ml}$ of CNTs in isopropanol, which was sonicated for $40 \mathrm{~min}$ to form homogeneous dispersions then spin-coated on top of the ITO glass substrate at a speed rate of $900 \mathrm{rpm}$ for $18 \mathrm{~s}$ and then $4000 \mathrm{rpm}$ for $60 \mathrm{~s}$, and then removed the residual solvent in a vacuum oven at $60{ }^{\circ} \mathrm{C}$ at a pressure of $100 \mathrm{~Pa}$ for $8 \mathrm{~h}$. The thickness of the resultant EMAR+CNTs composite film is $82 \pm 5 \mathrm{~nm}$. The top Al electrode layer of about $300 \mathrm{~nm}$ thickness was thermally evaporated through the shadow mask at a base pressure of $1.0 \times 10^{-4}$ Pa with a uniform deposition rate of 3-5 $\AA /$ s. The diameter of the top Al electrode is $200 \mu \mathrm{m}$. The electrical characterization of the memory device was performed by a Keithley 4200-SCS semiconductor parameter analyzer in an ambient atmosphere using a probe station at room temperature without any encapsulation. A two-terminal $I$ - $V$ test was carried out. The bottom electrode (ITO) was grounded during all the electrical measurement. Probe tip tightly contact with top electrode pressing the composite film will lead to the thickness reducing of active 
layer, and even cause the damage of the device. Although the thick top electrodes can effectively avoid the above situations occur, light touch between probe tip/top electrode contact is essential for ensuring the reliability and stability of performance tests. Structural formulas of EMAR and the structure of memory device are shown in Fig. 1a. Fig. 1b-d shows TEM image of the EMAR+CNTs mixture containing different content of CNTs. The TEM images manifest that the CNTs are randomly dispersed in the EMAR layer. The results showed that the two composites were well mixed.<smiles>C=C(C)OC(=O)OCC(O)COc1ccc(C(C)(C)c2ccc(OCC(O)COc3ccc(C(C)(C)c4ccc(OCC(O)C(=O)OC(C)=O)cc4)cc3)cc2)cc1</smiles>

(a)
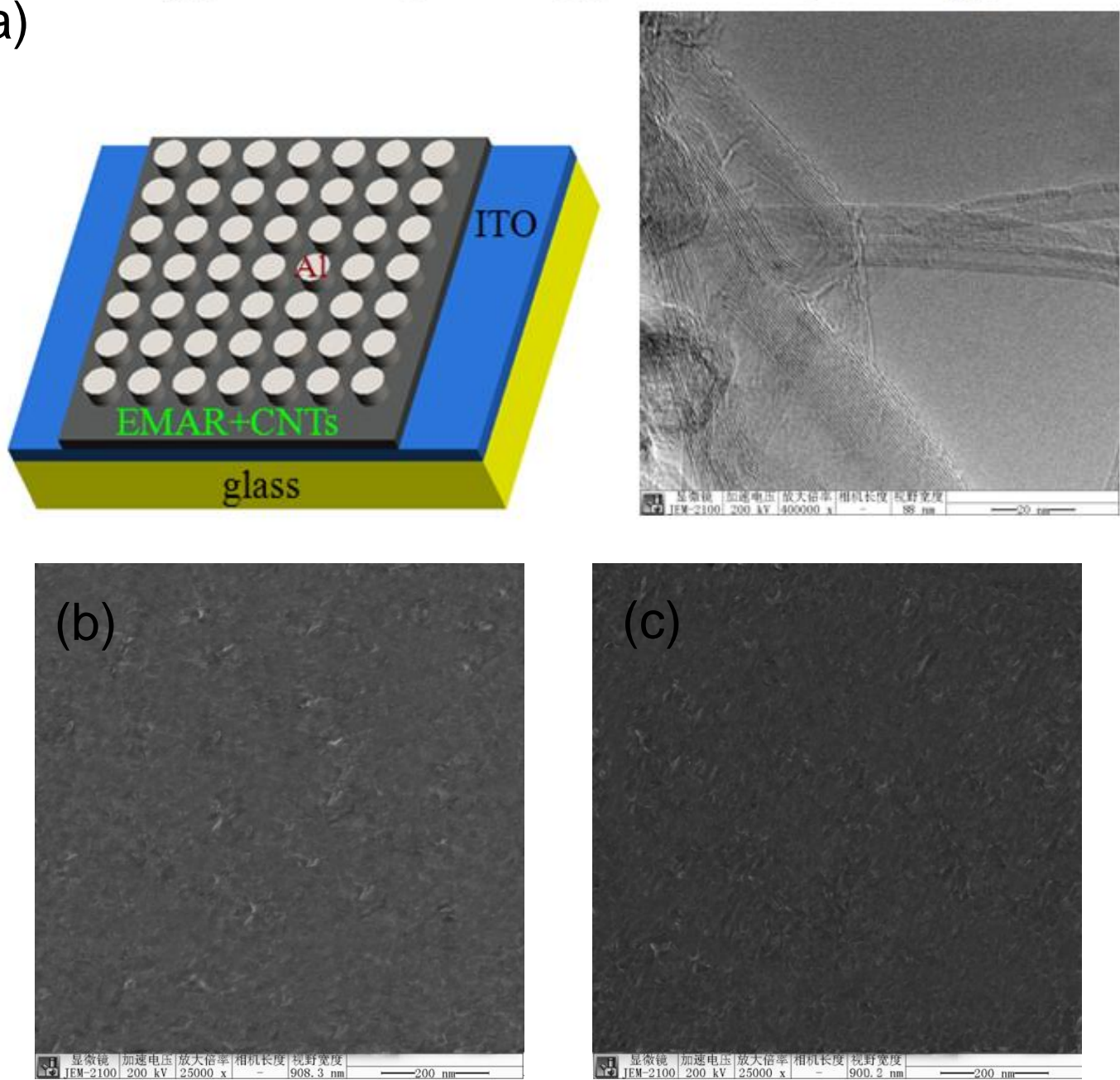


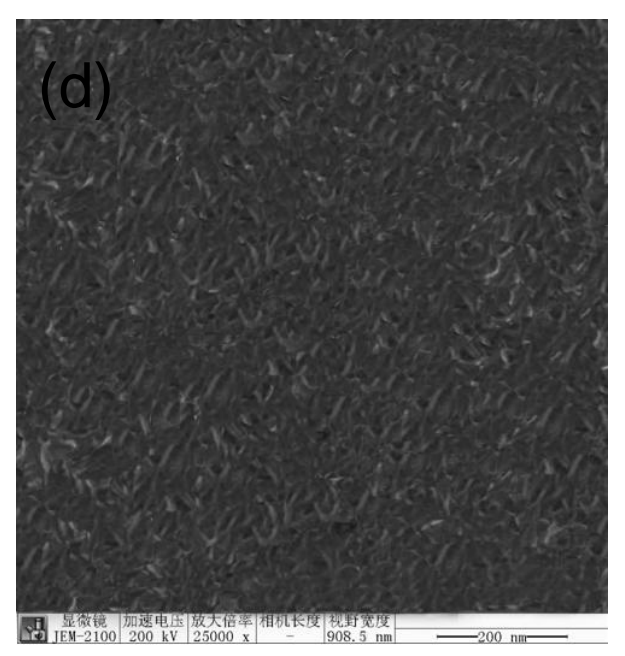

Fig. 1. (a) Structural formulas of EMAR and schematic diagram of the ITO/EMAR+CNTs/Al sandwich devices. The inset shows the TEM image of CNTs. TEM images of the EMAR+CNTs composite film containing (b) $0.8 \%$, (c) $2.3 \%$, and (d) $4 \%$ CNTs.

\section{Results and discussion}

The typical memory effect of the ITO/EMAR+CNTs/Al sandwich structure containing $0.8 \%$ CNTs is demonstated by the current-voltage $(I-V)$ characteristics. As presented in Fig. 2a, the ITO/EMAR+CNTs/Al device showed stable triple state memory characteristics (i.e., low resistance state (LRS), high resistance state (HRS) and intermediate resistance state (IRS)). The multilevel memory devices store more than 2-bits in a single cell, made it possible to achieve a high density memory.

Voltage was swept from 0 to $-6 \mathrm{~V}, 0$ to $-6 \mathrm{~V}, 0$ to $6 \mathrm{~V}$, and 0 to $6 \mathrm{~V}$ in the steps of $0.05 \mathrm{~V}$. Initially the device was in HRS. As the negative voltage applied to the top electrode was increased gradually (sweep 1), the current also increased. Two abrupt current changes can be clearly observed, each followed by a stable conductance plateau. One is at $-0.95 \mathrm{~V}$, the current suddenly jumped from $1.7 \times 10^{-5}$ to $2.5 \times 10^{-4} \mathrm{~A}$, followed by a plateau at $4.2 \times 10^{-4} \mathrm{~A}$ after an initial increase. Similarly, another is at $-2.15 \mathrm{~V}$, the current was switched from $4.2 \times 10^{-4}$ to $4.5 \times 10^{-2} \mathrm{~A}$ followed by a current platform. This is referred to as SET process and the device is known as to be in ON state. The occurrence of SET process for more than once, 
indicated the presence of more than one inherent LRS in the device. After the device reached its final LRS, there was no much increase in current with the increasing of applied voltage. The LRS of device is maintained in sweep 2. When the positive voltage was applied to the top electrode, device was still in LRS. Similar behavior was also observed in RESET process. As the increasing of voltage (sweep 3), there was a sudden drop in the current from $9.3 \times 10^{-2}$ to $1.2 \times 10^{-3} \mathrm{~A}$ at $3.6 \mathrm{~V}$ followed by a current platform and device achieved its IRS. With further increase of positive voltage there was a sudden drop in current from $2.1 \times 10^{-4}$ to $2.3 \times 10^{-5} \mathrm{~A}$ at $5.2 \mathrm{~V}$ and device reached the final HRS. This is the multistage RESET process, where device goes to OFF state. This process was repeatable for number of cycles, which established the device as a nonvolatile memory with multilevel conductance states. The observation of inherently present multi-resistance states during switching process indicates that the device possesses multilevel switching characteristics. Fig. $2 b$ shows the ON/OFF state current ratio of the device as a function of the applied voltage. The current ratios for LRS/IRS and LRS/HRS were 98.3 (reading at $-1.5 \mathrm{~V}$ ) and $9.0 \times 10^{3}$ (reading at $0.5 \mathrm{~V}$ ), respectively. In order to investigate the uniformity of the resistive switching parameters, cyclic programming operations of the ITO/EMAR+CNTs/Al devices were performed. Reproducible memory $I-V$ loops of 50 consecutive cycles are shown in Fig. 2c, The $I-V$ characteristics of the ITO/EMAR+CNTs/Al device did not show a noticeable change after 50 consecutive cycles. When the top electrode was initially biased "+" relative to the bottom, the $I-V$ characteristic always display a linear. The device remains in HRS and is absence of the switching effect. 

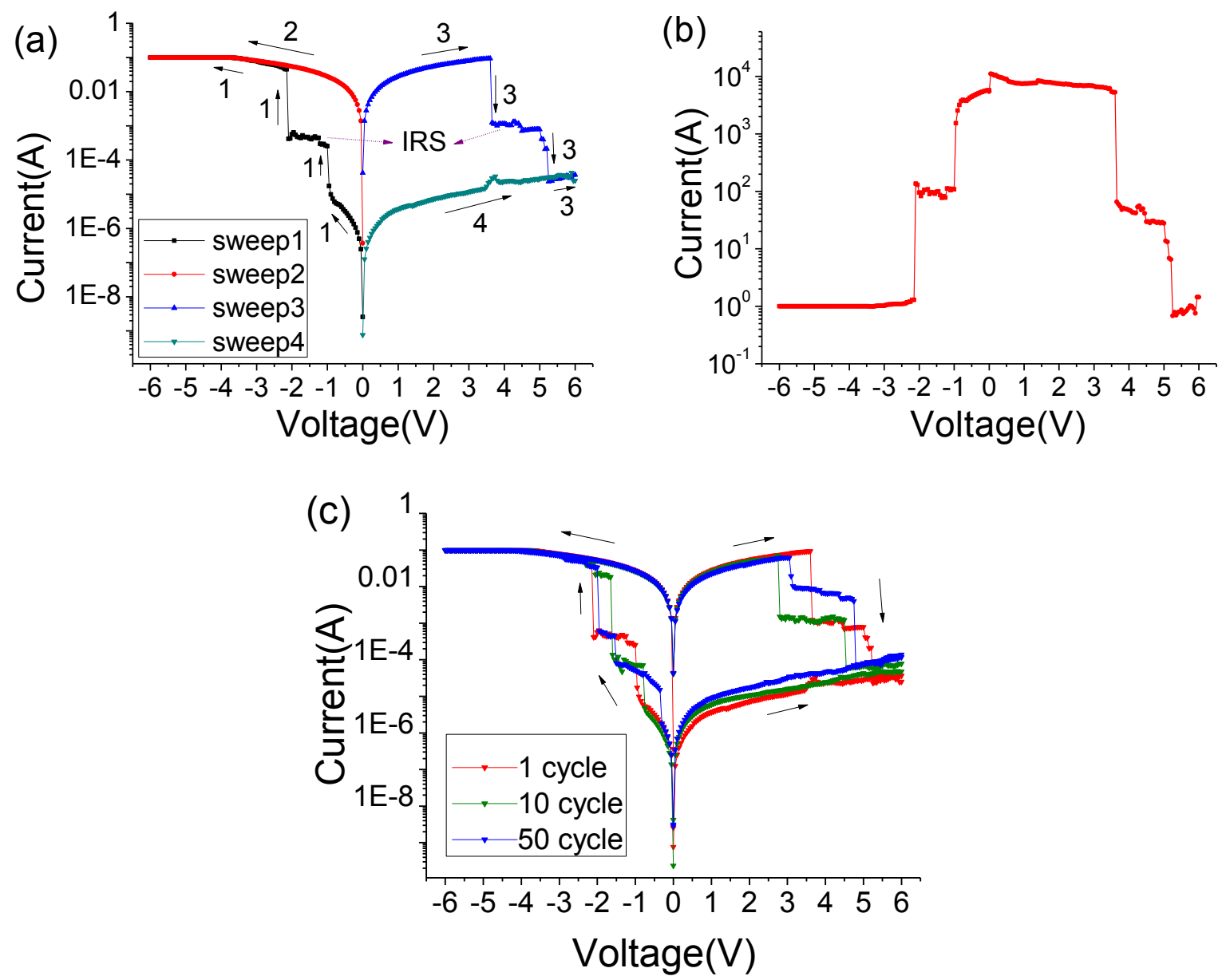

Fig. 2. (a) Typical $I-V$ characteristics of the ITO/EMAR+CNTs/Al device containing $0.8 \%$ CNTs. (b) ON/OFF state current ratio of the above device as a function of applied voltage. (c) Consecutive $I$ - $V$ cycles of ITO/EMAR+CNTs/Al device containing $0.8 \%$ CNTs.

To elucidate further the switching stability of the ITO/EMAR+CNTs/Al devices, cumulative probability distributions for $V_{\mathrm{SET}}$ and $V_{\mathrm{RESET}}$ are plotted in Fig. 3a. When the ITO/EMAR+CNTs/Al device was repeatedly switched between LRS and HRS, $V_{\text {RESET1 }}$ and $V_{\text {RESET2 }}$ distribute in a range of 2.60 to $4.05 \mathrm{~V}$ and 4.00 to $5.40 \mathrm{~V}$, respectively, while $V_{\mathrm{SET} 1}$ and $V_{\mathrm{SET} 2}$ show a distribution of -0.35 to $-1.4 \mathrm{~V}$ and -1.5 to $-3.15 \mathrm{~V}$, respectively. The overlapping window of operational voltages between the $V_{\text {RESET1 }}$ and $V_{\text {RESET2 }}$ and the small separation between $V_{\mathrm{SET} 1}$ and $V_{\mathrm{SET} 2}$ are extremely unfavorable to the uniformity of erase/write/read operation, it must be improved in the future to enable practical application in electronics. The statistical analysis also yields the average values of $V_{\mathrm{SET} 1}, V_{\mathrm{SET} 2}, V_{\mathrm{RESET} 1}$, and 
$V_{\text {RESET2 }}$ as $-0.8,-2.2,3.4$ and $4.8 \mathrm{~V}$, respectively. Fig. $3 \mathrm{~b}$ shows the resistance distributions of the LRS, IRS and HRS, respectively. As shown, the distribution ranges $R_{\mathrm{LRS}}, R_{\mathrm{IRS}}$ and $R_{\mathrm{HRS}}$ of ITO/EMAR+CNTs/Al devices are from 35.7 to $48.0 \Omega, 2.78 \times 10^{3}$ to $8.75 \times 10^{3} \Omega$, and $1.12 \times 10^{5}$ to $3.97 \times 10^{5} \Omega$, respectively. Different states can be clearly distinguished.

(a)

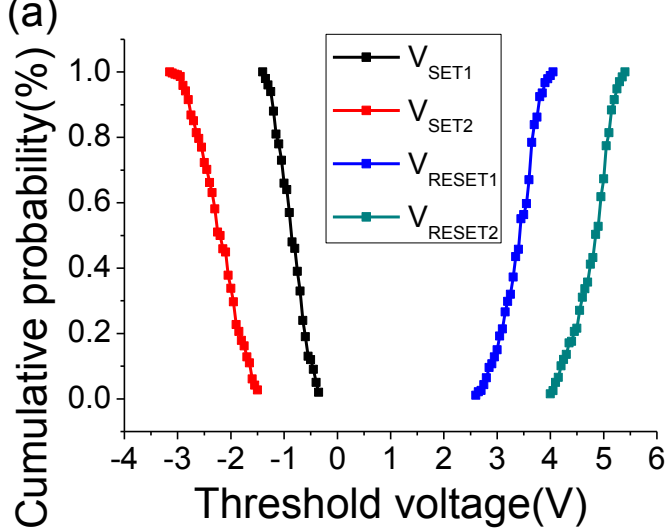

(b)

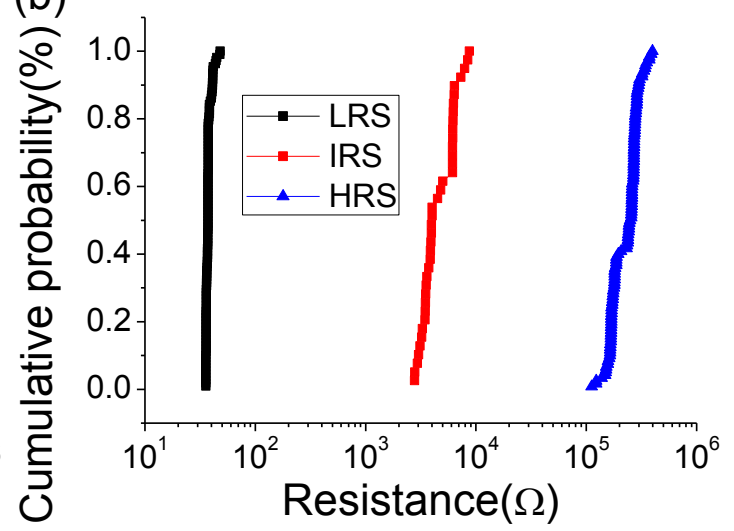

Fig. 3. (a) The statistical distributions of the threshold voltages of ITO/EMAR+CNTs/Al memory device containing $0.8 \%$ CNTs. (b) The statistical distributions of the resistances of ITO/EMAR+CNTs/Al memory device containing $0.8 \%$ CNTs.

Another key performance indicator for a memory device is the switching time [23]. Fig. 4a shows the transient response of the memory device. When a read pulse of $-4 \mathrm{~V}$ (above the threshold voltage $V_{\mathrm{SET} 2}$ ) was applied, the device was immediately turned to IRS from its initial HRS and then turned to LRS, and no distinguishable electrical delay was detected within the resolution of the Keithley 4200 (upper limit of the pulse rising time: $20 \mathrm{~ns}$ ). As shown in Fig. 4a, the transition time from HRS to IRS and from IRS to LRS for the ITO/EMAR+CNTs/Al device are 280 and $300 \mathrm{ns,}$, respectively. In order to investigate the switching characteristics and the rewritable nonvolatile memory of the ITO/EMAR+CNTs/Al device, write-read-erase-read (WRER) sequence measurements were performed. The writing, reading, erasing and rereading voltages for the $I-V$ characteristics were set as $-3.5,-0.3,5.5$ and $2 \mathrm{~V}$, respectively, as shown in Fig. 4b. The duration of each WRER cycle is $16 \mathrm{~s}$, and the current with the absolute values is measured for 2 points within each voltage level. Fig. 4c 
shows the corresponding current response of the device. The LRS current at a reading voltage of $-0.3 \mathrm{~V}$ is approximately $10^{-3} \mathrm{~A}$, and the HRS current at a reading voltage of $2 \mathrm{~V}$ is between $10^{-6}$ and $10^{-5}$ A. Switching failure in some points seems to be attributed to the overlapping window of operational voltages between the $V_{\text {RESET1 }}$ and $V_{\text {RESET2 }}$ and the transient response of the device current. These WRER switching cycles have shown repeatability for memory storage.
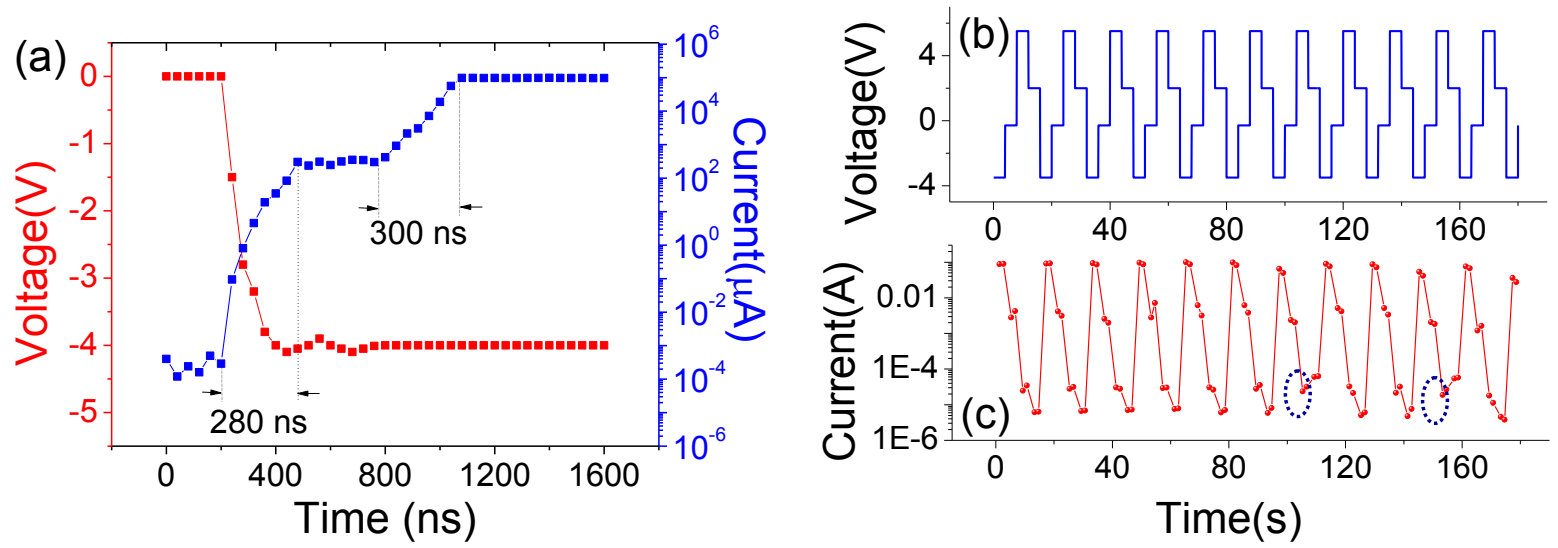

Fig. 4. (a) The transient response of ITO/EMAR+CNTs/Al memory device. Switching of the WRER sequence operation for the ITO/EMAR+CNTs/Al device: (b) input voltage and (c) output current as functions of time.

To understand conduction mechanism of the ITO/EMAR+CNTs/Al device, we have redrawn $I-V$ curves in three different resistance states. The log-log scale of the $I$ - $V$ curve (Fig. 5a) at the HRS state shows space charge limited conduction (SCLC) mechanism, consisting of an Ohmic current region (slope of 1.15) at low bias and a Mott-Gurney region (slope of 6.00) at high bias. During SCLC, with increasing applied voltage more and more charge carriers are injected to the active layer and therefore space charges are formed in active layer near the interface, resulting in space charge limited conduction. For the IRS state, $I-V$ graph in this region was re-plotted as shown in Fig. 5b. The negative value of slope with well fitted $\ln \left(I / V^{2}\right)$ versus $1 / V$ graph shows that the Fowler-Nordheim $(\mathrm{F}-\mathrm{N})$ tunneling mechanism was suited. Moreover, for the LRS of the device, fitting of the $I-V$ plot showed linear behavior of the device as shown in Fig. 5c indicating Ohmic conduction having slope $\sim 1$. 

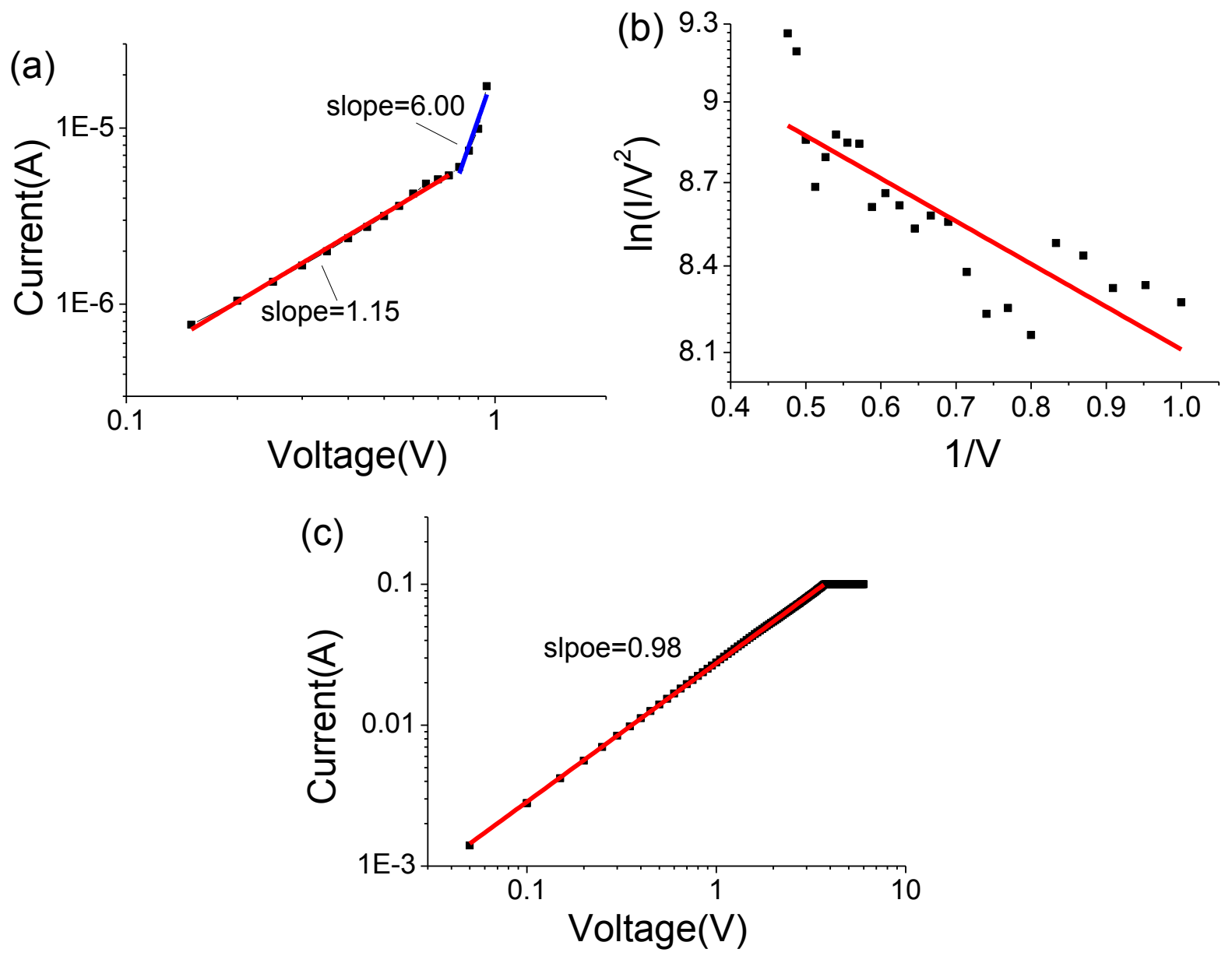

Fig. 5. $I-V$ graphs in log-log scale showing conduction mechanism for switching in ITO/EMAR+CNTs/Al memory device. (a) SCLC mechanism in HRS. (b) F-N tunneling mechanism in IRS. (c) Ohmic behavior in LRS.

To identify the physical nature of the resistive mechanism in the memory device, resistances in LRS and HRS were measured as a function of temperature. In LRS, the resistance of the device was found to increase linearly with increasing temperature in the range of 296-383 K as can be seen in Fig. 6a, indicating metallic behavior. The observed linear temperature dependence of metallic behavior in LRS could be fitted by the following equation; $R(T)=R_{0}\left[1+\alpha\left(T-T_{0}\right)\right]$, where $R_{0}$ is the resistance at temperature $T_{0}, \alpha$ is the temperature coefficient of resistance, and $R(T)$ is the resistance at temperature $T$. From the fitting of the experimental data, $\alpha$ was estimated to be $\sim 4.2 \times 10^{-3}$, which is typical for conduction in metallic nanowires indicating presence of metal like filaments in the device 
between the top and bottom electrodes.

In contrary to metallic behavior in LRS, the device in HRS showed semiconducting behavior as depicted in Fig. 6b. The resistance of the device decreased with increasing temperature in HRS following the Arrhenius law given by: $R(T)=R_{\mathrm{A}} \exp \left(E_{\mathrm{AC}} / K T\right)$, where $R_{\mathrm{A}}$ is the Arrhenius pre-exponential factor for resistance, $E_{\mathrm{AC}}$ is the activation energy for conduction, and $K$ is the Boltzmann constant. The inset in Fig. 6b shows the plot of $\ln (R)$ as a function of $1 / K T$, in which the slope of a fitted line is actually the activation energy. From the fitting of this linear relationship, the $E_{\mathrm{AC}}$ was estimated to be $\sim 20.2 \mathrm{meV}$.
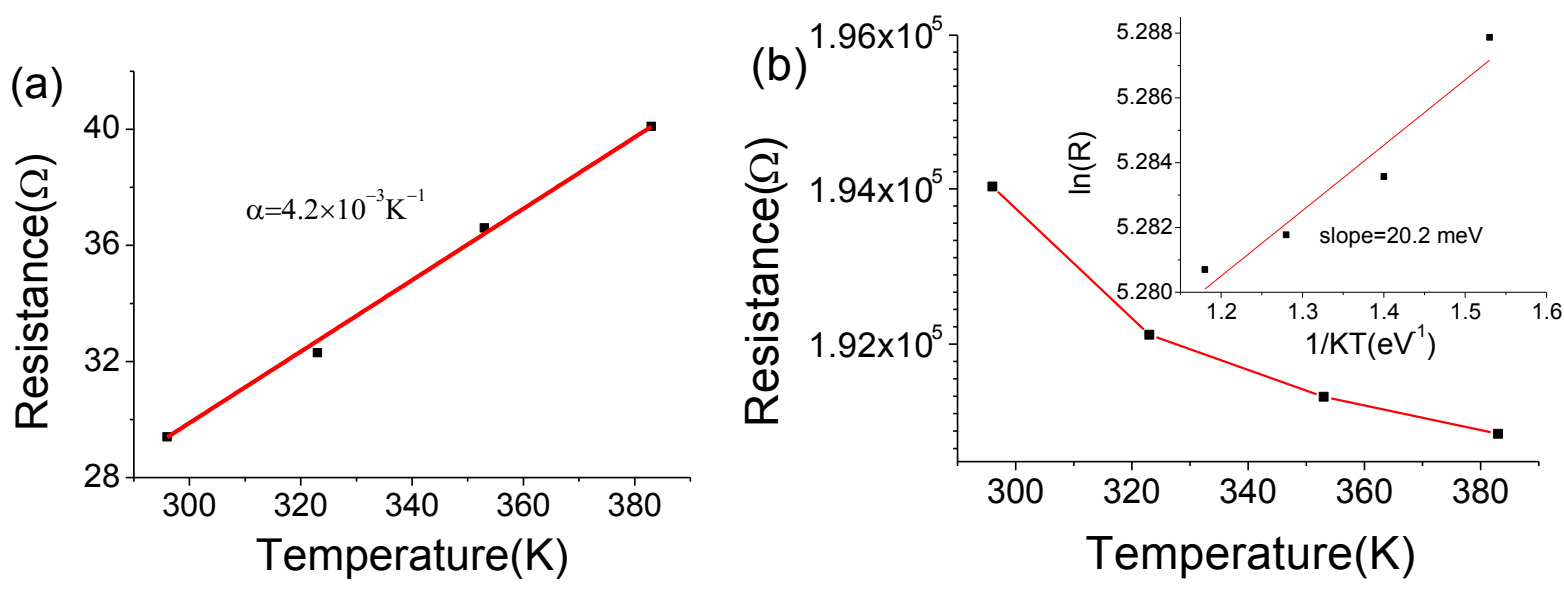

Fig. 6. (a) Temperature dependence of resistances in LRS of the device. (b) Temperature dependence of resistances in HRS of the device.

Many findings suggest that the resistive switching is related to formation of a thin aluminum oxide layer at $\mathrm{Al}$ top electrode [38,42-45]. For this reason the $\mathrm{Al}$ top electrode is substituted by a $\mathrm{Ag}$ top electrode, because the work functions of $\mathrm{Ag}$ is similar to that of $\mathrm{Al}$, and Ag has greater inertia from oxidation than Al. Our results suggest that there is a similar switching properties to that in the devices using $\mathrm{Al}$ top electrode, and no significant change in the switching behaviors, and only changes in the write/erase voltages and current of HRS were found, so that the switching effect observed here cannot due to the aluminum oxide layer between the $\mathrm{Al}$ top electrode and active layer. This results indicate that the organic layer 
take part in the conduction.

The conductance switching mechanism of ITO/EMAR+CNTs/Al device can be understood by trapping and detrapping of electrons in CNTs [25,35,46,47]. Furthermore, presence of various types of defects in CNTs is also a well known fact [48-50]. It is important to note that the defects/trapping sites present in CNTs may have different potentials [48-50]. When the negative bias is applied on the top electrode, the electrons are injected to the active layer, and conduction started following SCLC mechanism as discussed above in the switching process. With further increase in voltage, the electrons injected are captured by the electron trap nodes present in CNTs. Once majority of the trapping sites in CNTs are occupied, they are capable of generating a local internal field and these trapped sites filled with carriers form a conducting path in the EMAR matrix, as a result of which current increases abruptly with the increasing of applied voltage and switches device from HRS to IRS. The IRS was stabilized by the selective electron trap in CNTs with a shallow level. In this case, as discussed above the current conduction follows F-N tunneling. The multistage SET process in our device may be ascribed to the formation of multi-channels with different threshold potentials of trapping sites in CNTs. These trapping sites remain occupied by electrons even though applied voltage is reduced to zero, hence maintaining the LRS of the device leading to nonvolatility. Further changing the bias polarity into the positive voltage causes the current to increase with increasing voltage because of the presence of trapped charge carriers in the nodes. When a given positive voltage is reached, current starts to decrease due to the de-trapping of electrons from the CNTs which further results in breaking of conducting paths. The electrons trapped in CNTs were selectively released at a low 
positive voltage (3.65 V) (Fig. 2a). As a result, the device transited from LRS to IRS and sustained the IRS. At another higher given applied bias, when majority of electrons trapped in charge nodes are ejected back to electrode, the conducting channels are disrupted then the device is transformed to HRS. However, after RESET process with the smaller positive voltage bias, the HRS current does not decrease to the much lower value. It implies that there are a few residual unruptured conducting filaments within the memory film where the current could flow through. The occurring of multistage RESET process has the same reason as discussed above in SET process. In short, it may be ascribed to the rupture of multi-channels with different potentials. Presence of the intermediate stage exhibiting multilevel behavior is due to formation of multi-filaments [51,41] and with different threshold potential [52,41]. As above, CNTs provides trapping sites and nodes for charge trapping, the switching characteristics of device are credited to the presence of CNTs. Above all, it becomes obvious that the density of electrons trapped in CNTs and the extent of formation of conducting channels in the device can be controlled by limiting the current flowing through the device. This might render the device in different LRS bringing about tuned multi-bit storage capability.

Based on the above analysis we have applied different values of compliance current varying from $1 \mathrm{~mA}$ to $100 \mathrm{~mA}$ and correspondingly obtained different LRS as shown in Fig. 7a. When compliance currents $\left(I_{\mathrm{CC}}\right)$ of 1,10 , and $100 \mathrm{~mA}$ are applied, it can be seen that the HRS resistance $\left(R_{\mathrm{HRS}}\right)$ is basically independent of $I_{\mathrm{CC}}$, and the LRS resistance $\left(R_{\mathrm{LRS}}\right)$ increases with the decreasing of $I_{\mathrm{CC}}$. As a consequence, the device showed well distinguished four states including one HRS and three LRS. The three LRS observed by imposing compliance current of 1, 10 and $100 \mathrm{~mA}$ are defined as LRS1, LRS2 and LRS3 respectively. 
It was clear that the higher compliance current imposed on the device, the higher LRS current was observed, and the LRS is determined by the magnitude of applied compliance current. Hence, it can be imagined that the maximum allowed current (compliance current) influences the formation of percolating filaments. Because stronger or more conducting filaments are expected to be induced by higher compliance current. Therefore, the compliance current is a key parameter which influences the value of the LRS current. Therefore, the multilevel LRS are achieved in ITO/EMAR+CNTs/Al memory device by setting different $I_{\mathrm{CC}}$ values.

Fig. $7 \mathrm{~b}$ shows the compliance current dependence on the resistances of both LRS and HRS. The resistance values were measured at $2 \mathrm{~V}$. LRS resistance decreased with increasing the compliance current. The LRS/HRS resistance ratio increased from 24 to 7500 when increasing the compliance current from 1 to $100 \mathrm{~mA}$ at $2 \mathrm{~V}$. The inset in Fig. $7 \mathrm{~b}$ shows the relationship between the compliance current and the SET voltage. SET voltage increased with the increasing of compliance current as shown in Fig. 7b. Due to the higher current state achieved in the memory device, it requires a higher switch-on voltage to generate the effective conducting filaments in order to switch the device to LRS.

By the application of high compliance current, most of the trapping sites were filled and as a consequence a large number of conductive paths for transport were formed, while in case of lower compliance current, a very limited number of trapping sites are filled generating relatively small density of conductive channels, resulting in higher resistance. Experimental results indicate that the presence of tuned multilevel conductance in ITO/EMAR+CNTs/Al device and make it possible for high density data storage.

To confirm the performance and stability of the ITO/EMAR+CNTs/Al device, its endurance and retention properties were tested. In the retention characteristics test as shown in Fig. 7c, the device stably maintained the initial resistance values of HRS, LRS1, LRS2 and LRS3 for almost $16 \mathrm{~h}$ under a constant $-0.3 \mathrm{~V}$ of read voltage. Here, we fixed the read voltage 
at $-0.3 \mathrm{~V}$ because it was not close to the threshold voltage and had the stable performance.

The resistance ratios for HRS/LRS1, LRS1/LRS2 and LRS2/LRS3 were 53.4, 10.1 and 10.7, respectively. Fig. 7d shows the endurance properties of the ITO/EMAR+CNTs/Al device under four different resistance states at $1.5 \mathrm{~V}$. The pulse period and pulse width are 2 and 1 $\mu \mathrm{s}$, respectively. The four different states in memory devices are stable for at least $6 \times 10^{4}$ continuous read cycles. The resistance ratios for HRS/LRS1, LRS1/LRS2 and LRS2/LRS3 were 25.0, 11.6 and 10.8, respectively. The result reveals well distinguished resistance states of HRS, and three LRS having almost constant ratio and with no degradation over the time. These results provide the device as a promising candidate for high density storage devices with excellent performance and good stability.
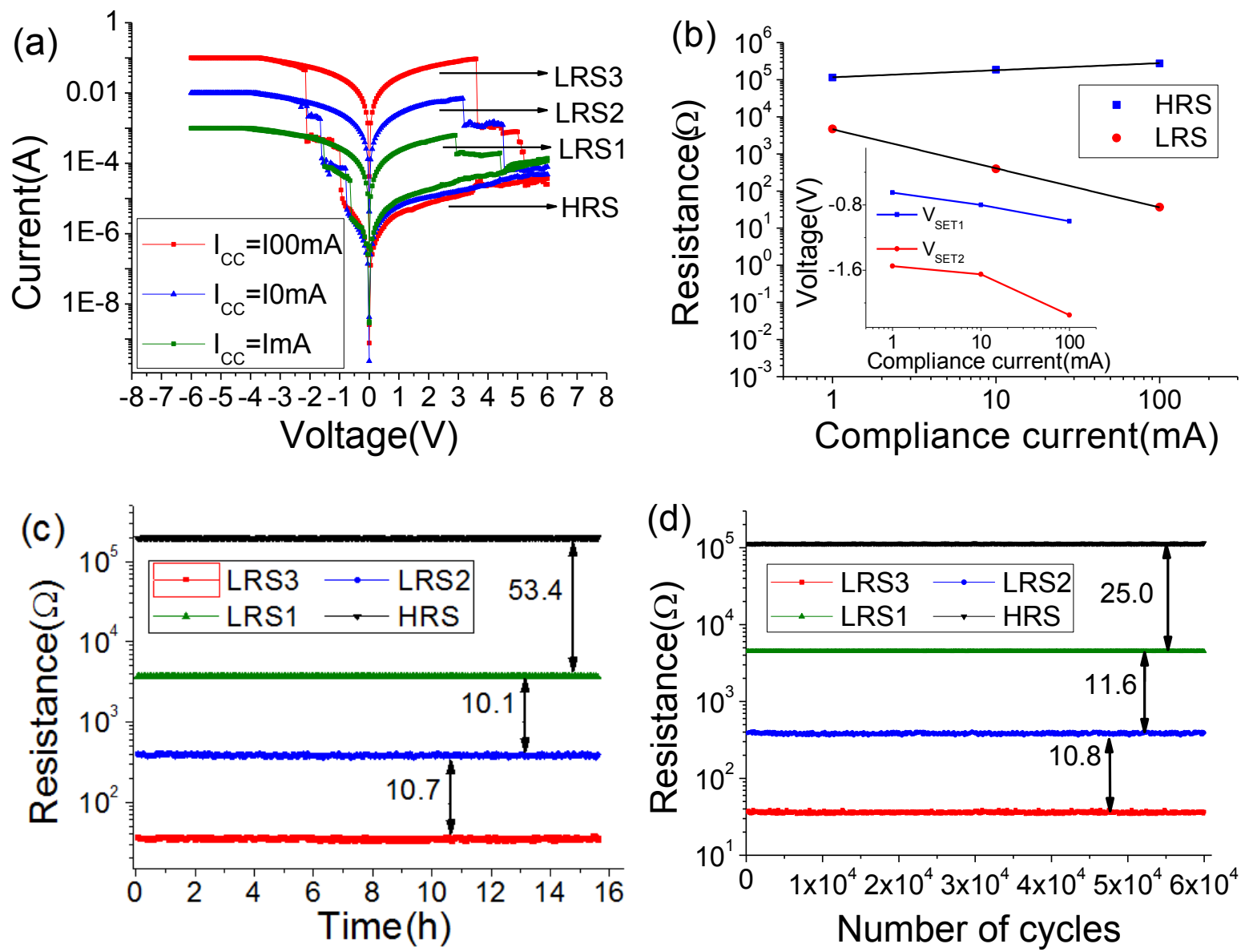

Fig. 7. (a) $I-V$ characteristics for ITO/EMAR+CNTs/Al device under different compliance current from 1 to $100 \mathrm{~mA}$. (b) Compliance current dependence of the resistance. Resistances were read at $2 \mathrm{~V}$ in all LRS and HRS. The inset shows the relationship between the compliance current and the SET voltage. (c) 
Retention characteristics of ITO/EMAR+CNTs/Al device with reading voltage of $-0.3 \mathrm{~V}$ for all four states.

(d) Endurance properties of ITO/EMAR+CNTs/Al device under four different resistance state. The reading voltage is $1.5 \mathrm{~V}$.

In addition to obtain multilevel LRS in the ITO/EMAR+CNTs/Al device by changing the compliance current, the multilevel HRS can also be performed by employing different concentration of CNTs in composite film, as shown in Fig. 8a. We investigated and compared the resistive memory characteristics with various CNTs compositions $(0.8-4.0$ wt \%). Due to the fine dispersibility, all the devices exhibited reliable rewritable resistive memory characteristics as shown in Fig. 8a. The device showed well distinguished four states including one LRS and three HRS. By changing the content of CNTs, three HRS could be observed, which are defined as HRS1, HRS2 and HRS3 respectively. Compared with Fig. 7a, the current of LRS is almost unchanged, but the current of HRS increases with increasing CNTs content from 0.8 to $4.0 \mathrm{wt} \%$, leading to the tuned resistance ratio between the LRS and HRS. The increase of HRS3 current at $4.0 \mathrm{wt} \%$ is attributed to the initiation of the partial percolation of CNTs. This variation of resistive switching behavior with CNTs composition also supports that well-dispersed CNTs indeed played the part of charge trap in the EMAR matrix.

The influence of CNTs content in the composite films on device performance, including HRS current under a given voltage $(2 \mathrm{~V})$ and ON/OFF state current ratio, is summarized in Fig. 8b. We note that the ON/OFF state current ratio increases by 2 orders of magnitude when CNTs content is decreased. The HRS current increased by 2 orders of magnitude when CNTs content is increased. The current was proportional to the charge trap density given by the CNTs composition. Furthermore, the average separation between neighboring CNTs should decrease with the increase of CNTs content. The electrons captured in a CNT could readily transfer to the neighboring CNTs (low value of resistance in HRS) if the separation between neighboring CNTs is close. From the results of Fig. 7a and 8a, as a result, not only the 
multilevel LRS but the multilevel HRS can be obtained in this work. It implies the possibility for the multilevel storage by an appropriate operation of the ITO/EMAR+CNTs/Al device.
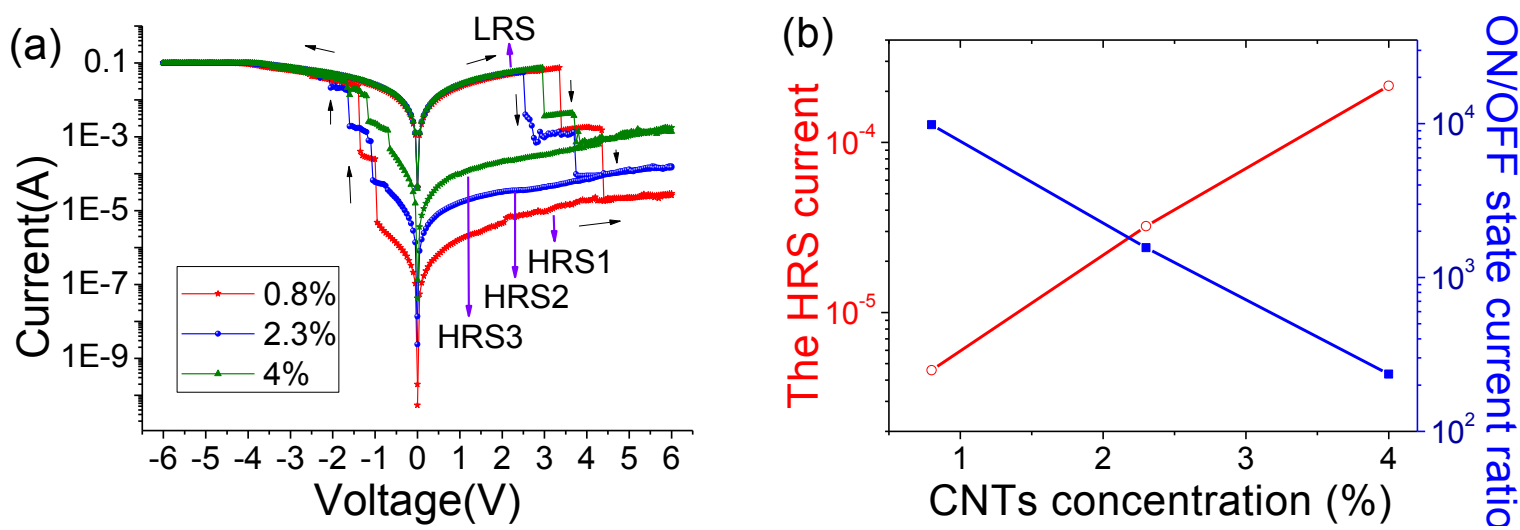

Fig. 8. (a) $I-V$ characteristics of ITO/EMAR+CNTs/Al devices under various CNTs contents. (b) The influence of CNTs content on HRS current under a given voltage $(2 \mathrm{~V})$ and ON/OFF state current ratio.

In order to consider the capability for multilevel capability application, measurement of retention characteristic at various electrical states of ITO/EMAR+CNTs/Al device containing different content of CNTs is performed. As shown in Fig. 9a, the retention time was extended with the increasing of CNTs content. It may be ascribed to the formation of stable filament formation in EMAR matrix correspondence to a higher content of CNTs. The endurance performance at various electrical states obtained for ITO/EMAR+CNTs/Al device is presented in Fig. 9b. During pulse cycles (The pulse period and pulse width are 2 and $1 \mu \mathrm{s}$, respectively), HRS3 (containing 4\% CNTs) is long, and HRS1 (containing $0.8 \%$ CNTs) is short. The endurance performance is also related to the content of CNTs, which may be due to the varying filling degrees of traps in the active layer with different content of CNTs. The different conduction states remain at ambient conditions and the clearly distinguished states indicate the excellent stability of the device. 

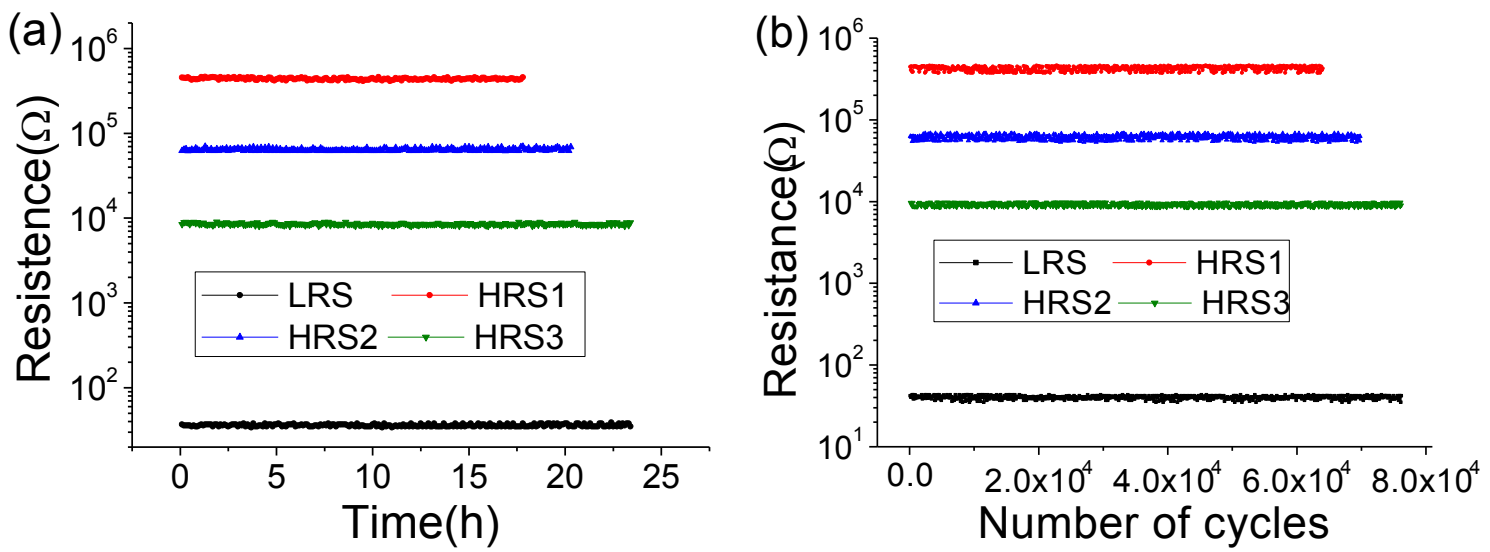

Fig. 9. (a) Retention characteristics of the ITO/EMAR+CNTs/Al device with reading voltage of $2 \mathrm{~V}$ for all four states. (b) Endurance properties of the ITO/EMAR+CNTs/Al device under four different resistance state. Resistances for all four states were read at $2 \mathrm{~V}$.

Therefore, by proper controlling compliance current and content of CNTs in EMAR matrix, the multilevel LRS and even the multilevel HRS can be achieved in the ITO/EMAR+CNTs/Al device. All the multilevel LRS and HRS can be read without affecting the stored information. Owing to all the important properties as mentioned above, the ITO/EMAR+CNTs/Al device is proposed as a good promising candidate for the multilevel capability application in the next generation nonvolatile memory.

\section{Conclusion}

In summary, the multilevel LRS as well as the multilevel HRS are demonstrated for data storage in the ITO/ EMAR+CNTs/Al device. The effects of the compliance current and the content of CNTs in composite film on the multilevel resistive switching properties are also investigated. Multilevel resistance states with stable intermediate resistance states were found to be inherently present in these devices. Based on the experimental results, all the multilevel memory states are distinguishable and exhibit good endurance and retention characteristics with stable resistances. This structure fabricated by simple spin coating method offers a promising and reliable design scheme for the low cost solution processing of multilevel 
resistive memory and high density storage media.

\section{Acknowledgements}

The authors are grateful to the support of the National Science Foundation of China (Grant no. 61204127 and 21372067), Doctoral Fund of Ministry of Education of China (20132301110001).

\section{References}

[1] B. Cho, S. Song, Y. Ji, T.-W. Kim, T. Lee, Adv. Funct. Mater. 21 (2011) 2806.

[2] H. J. Yen, C.-J. Chen, G. -S. Liou, Adv. Funct. Mater. 23 (2013) 5307.

[3] A.-D. Yu, T. Kurosawa, Y.-H. Chou, K. Aoyagi, Y. Shoji, T. Higashihara, M. Ueda, C.-L. Liu, W.-C. Chen, ACS Appl. Mater. Interfaces 5 (2013) 4921.

[4] Q. D. Ling, D.-J. Liaw, C. X. Zhu, DS.-H. Chan, E.-T. Kang, K.-G. Neoh, Prog. Polym. Sci. 33 (2008) 917.

[5] L.-C. Lin, H.-J. Yen, C.-J. Chen, C.-L. Tsai, G.-S. Liou, Chem. Commun. 50 (2014) 13917.

[6] G. Li, K. Zheng, C. Wang, K. S. Leck, F. Hu, X. W. Sun, Q. Zhang, ACS Appl. Mater. Interfaces 5 (2013) 6458.

[7] Y.-G. Ko, D. M. Kim, K. Kim, S. Jung, D. Wi, T. Michinobu, M. Ree, ACS Appl. Mater. Interfaces 6 (2014) 8415.

[8] M. A. Mamo, W. S. Machado, W. A. L. v. Otterlo, N. J. Coville, I. A. Hümmelgen, Org. Electron. 11 (2010) 1858.

[9] U. S. Bhansali, M. A. Khan, D. Cha, M. N. AlMadhoun, R. Li, L. Chen, A. Amassian, I. N. Odeh, H. N. Alshareef, ACS Nano 7 (2013) 10518.

[10] C. J. Chen, H. J. Yen, W. C. Chen, G. S. Liou, J. Mater. Chem. 22 (2012) 14085. 
[11] H. Wang, F. B. Meng, B. W. Zhu, W. R. Leow, Y. Q. Liu, X. D. Chen, Adv. Mater. (2015) DOI: $10.1002 /$ adma.201405728.

[12] A. Rani, J. M. Song, M. J. Lee, J. S. Lee, Appl. Phys. Lett. 101 (2012) 233308.

[13] S. R. Forrest, Nature 428 (2004) 911.

[14] J. H. Wu, Y H. J. en, Y. C. Hu, G. S. Liou, Chem. Commun. 50 (2014) 4915.

[15] C. J. Chen, Y. C. Hu, G. S. Liou, Chem. Commun. 49 (2013) 2536.

[16] A. Suresh, G. Krishnakumar, M. A. G. Namboothiry, Phys. Chem. Chem. Phys. 16 (2014) 13074.

[17] W. S. Ren, Y. X. Zhu, J. F. Ge, X. F. Xu, R. Sun, N. J. Li, H. Li, Q. F. Xu, J. W. Zheng, J. M. Lu, Phys. Chem. Chem. Phys. 15 (2013) 9212.

[18] S. M. Jilani, T. D. Gamot, P. Banerji, S. Chakraborty, Carbon 64 (2013) 187.

[19] J. Q. Liu, Z. Y. Yin, X. H. Cao, F. Zhao, L. H. Wang, W. Huang, H. Zhang, Adv. Mater. 25 (2013) 233.

[20] G. Khurana, P. Misra, R. S. Katiyar, J. Appl. Phys. 114 (2013) 124508.

[21] H. Y. Jeong, J. Y. Kim, J. W. Kim, J. O. Hwang, J. -E. Kim, J. Y. Lee, T. H. Yoon, B. J. Cho, S. O. Kim, R. S. Ruoff, S.-Y. Choi, Nano Lett. 10 (2010) 4381.

[22] H. Wang, Y. M. Du, Y. T. Li, B. W. Zhu, W. R. Leow, Y. G. Li, J. S. Pan, T. Wu, X. D. Chen, Adv. Funct. Mater. 25 (2015) 3825.

[23] W. S. Machado, M. A. Mamo, N. J. Coville, I. A. Hümmelgen, Thin Solid Films 520 (2012) 4427.

[24] C. Q. Ye, Q. Peng, M. Z. Li, J. Luo, Z. M. Tang, J. Pei, J. M. Chen, Z. G. Shuai, L. Jiang, Y. L. Song, J. Am. Chem. Soc. 134 (2012) 20053.

[25] S. K. Hwang, J. M. Lee, S. Kim, J. S. Park, H. I. Park, C. W. Ahn, K. J. Lee, T. Lee, S. O. Kim, Nano Lett. 12 (2012) 2217.

[26] B. L. Hu, X. J. Zhu, X. X. Chen, L. Pan, S. S. Peng, Y. Z. Wu, J. Shang, G. Liu, Q. Yan, 
R.-W. Li, J. Am. Chem. Soc. 134 (2012) 17408.

[27] P.-Y. Gu, F. Zhou, J. K. Gao, G. Li, C. Y. Wang, Q. -F. Xu, Q. C. Zhang, J. -M. Lu, J. Am. Chem. Soc. 135 (2013) 14086.

[28] S. I. White, P. M. Vora, J. M. Kikkawa, K. I. Winey, Adv. Funct. Mater. 21 (2011) 233.

[29] D. Y. Yun, N. H. Lee, H. S. Kim, S. W. Lee, T. W. Kim, Appl. Phys. Lett. 104 (2014) 023304.

[30] T. Kondo, S. M. Lee, M. Malicki, B. Domercq, S. R. Marder, B. Kippelen, Adv. Funct. Mater. 18 (2008) 1112.

[31] S. Song, B. Cho, T. W. Kim, Y. Ji, M. Jo, G. Wang, M. Choe, Y. H. Kahng, H. Hwang, T. Lee, Adv. Mater. 22( 2010) 5048.

[32] C.-J. Chen, Y.-C. Hu, G. -S. Liou, Chem. Commun. 49 (2013) 2804.

[33] Q. Zhang, J. Pan, X. Yi, L. Li, S. Shang, Org. Electron. 13 (2012) 1289.

[34] M. A. Mamo, A. O. Sustaita, N. J. Coville, I. A. Hümmelgen, Org. Electron. 14 (2013) 175.

[35] G. Liu, Q. D. Ling, E. Y. H. Teo, C. X. Zhu, D. S. H. Chan, K. G. Neoh, E.-T. Kang, ACS Nano 3 (2009) 1929.

[36] W. T. Kim, J. H. Jung, T. W. Kim, Appl. Phys. Lett. 95 (2009) 022104.

[37] G. Liu, Q. D. Ling, E. T. Kang, K. G. Neoh, J. Appl. Phys. 102 (2007) 024502.

[38] J. A. Ávila-Niño, W. S. Machado, A. O. Sustaita, E. Segura-Cardenas, M. Reyes-Reyes, R. López-Sandoval, I. A. Hümmelgen, Org. Electron.13 (2012) 2582.

[39] S. ChandraKishore, A. Pandurangan, RSC Adv. 4 (2014) 9905.

[40] R. H. Baughman, A. A. Zakhidov, W. A. D. Heer, Science 297 (2002) 787.

[41] C. Wang, Z. X. Guo, S. Fu, W. Wu, D. Zhu, Prog. Polym. Sci. 29 (2004) 1079.

[42] M. Cölle, M. Büchel, D. M. d. Leeuw, Org. Electron. 7 (2006) 305.

[43] F. Verbakel, S. C. J. Meskers, R. A. J. Janssen, H. L. Gomes, M. Cölle, M. Büchel, D. M. 
d. Leeuw, Appl. Phys. Lett. 91(2007)192103 .

[44] S. Karthäuser, B. Lüssem, M. Weides, J. Appl. Phys. 100 (2006) 094504.

[45] T. Oyamada, H. Tanaka, K. Matsushige, H. Sasabe, C. Adachi, Appl. Phys. Lett. 83 (2003) 1252.

[46] G. Khurana, P. Misra, R. S. Katiyar, Carbon 76 (2014) 341.

[47] H. T. Lin, Z. Pei, Y. J. Chan, IEEE Electr. Device Lett. 28 (2007) 569.

[48] G.-D. Lee, C.-Z. Wang, E. Yoon, N. -M. Hwang, K. -M. Ho, Appl. Phys. Lett. 97 (2010) 093106.

[49] M. Ishigami, H. J. Choi, S. Aloni, S. G. Louie, M. L. Cohen, A. Zettl, Phys. Rev. Lett. $93(2004) 196803$.

[50] J. Kotakoski, A. V. Krasheninnikov, K. Nordlund, Phys. Rev. B 74 (2006) 245420.

[51] C. Li, H. Jiang, Q. Xia, Appl. Phys. Lett. 103 (2013) 062104.

[52] A. Younis, D. Chu, S. Li, Nanoscale Res. Lett. 8 (2013) 6918. 
The memory device based on EMAR+CNTs composite exhibits multilevel conductivity states with stable intermediate resistance states.

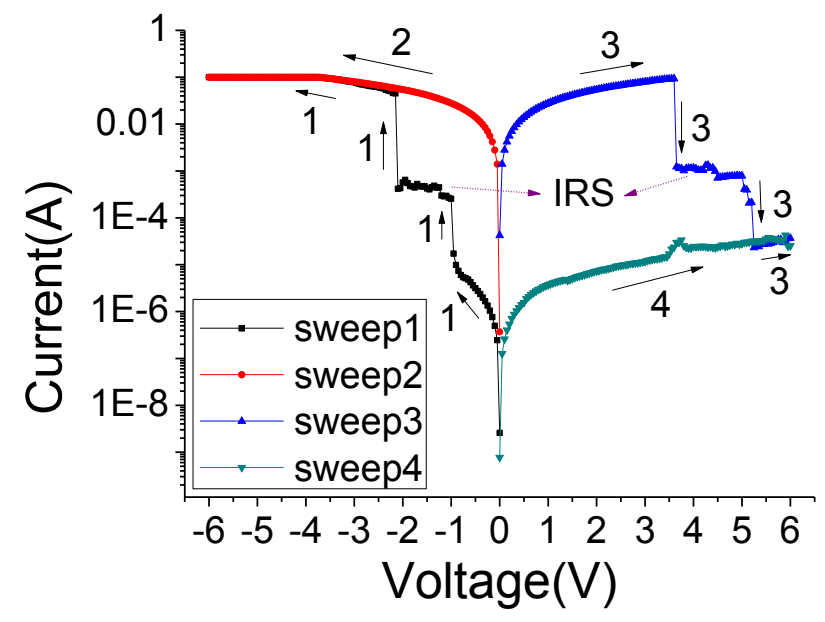

